\title{
Milling of Thin Walled Components from Aluminum Alloys by Considering the Conditions of Rigidity and Automation Assignment Elements of a Cutting Mode on CNC Machines
}

\author{
Michail Nazarov ${ }^{1}$ and Evgeny Kiselev ${ }^{1}$ \\ ${ }^{1}$ Ulyanovsk State Technical University, 432027, 32 Severny Venets Street, Ulyanovsk, Russian \\ Federation
}

\begin{abstract}
The work is devoted to the problems of manufacturing thinwalled parts of the aviation industry and instrument engineering on modern numerical control machines. The main reason of defects of such products loss of dimensional accuracy is revealed. The reason for this is the elastic deformation of the workpiece during milling. A mathematical model for calculating the elastic pressing of a thin wall during milling is proposed. The method of checking the adequacy of the developed model with the help of engineering analysis system is presented. The minimum set of geometrical parameters of the processed element and the cutting tool necessary for search of the rational cutting mode was defined. A new approach to automation of this process present through the evaluation of all possible solutions. The calculation of the rational cutting mode was made taking into account the conditions of rigidity of the workpiece. Conclusions on the efficiency of the developed technique are made.
\end{abstract}

\section{Introduction}

Currently, in the aviation industry and instrumentation a large number of parts are used, which are a complex combination of non-rigid elements (thin walls, panels). Their separate processing causes significant difficulties, and in combination with similar no rigid elements, makes this technology in accordance with the requirements of the drawing very costly and time-consuming. 


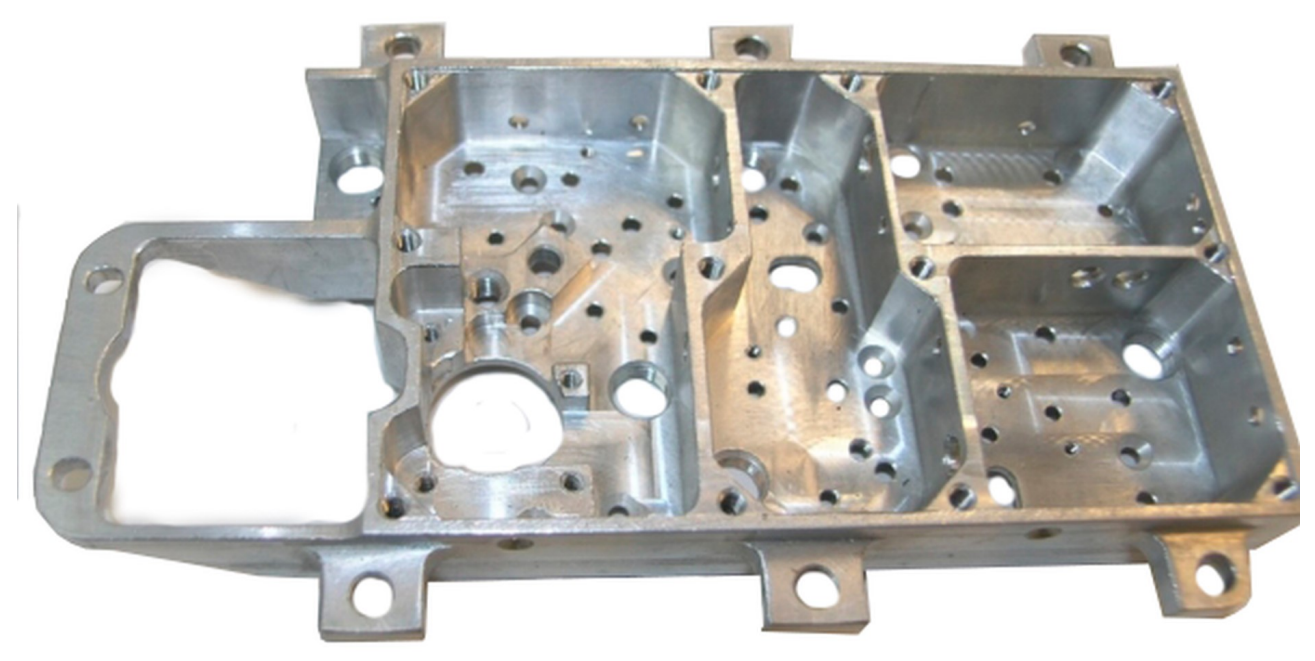

Fig. 1. Part consisting of a series of adjacent non-rigid elements.

The main problem in the processing is the elastic deformation of the walls in the milling process, which leads to vibration, shape deviation and going beyond of tolerance of the geometric dimensions of the processed element. The reason for this is the lack of recommendations from the manufacturer of the cutting tool for the processing of non-rigid blanks. Engineers and technologists can only access information about the maximum permissible milling modes, which are presented for an ideal, absolutely rigid technological system. $[1,2,3,4,5]$

The aim of this work is to develop an automated system assignment of the cutting conditions during milling of non-rigid workpieces of machine parts. To achieve the goal, it is necessary to solve the following tasks: to develop a mathematical model for determining the value of elastic deformations of the processed element in the milling process; to develop a technique for finding the most productive cutting mode that ensures the fulfillment of the requirements specified in the drawing.

\section{The description of the theoretical studies}

To implement this process, it is necessary to present the processed wall in the form of a plate length $l$, height $h$ and thickness $s$ fixed on three sides, which acts concentrated force Py. The radial component of the cutting force leads to a mutual elastic deformation of the workpiece and the cutting tool. Mills made of hard alloy have a much greater stiffness in comparison with thin walls of aluminum and titanium alloys used in the aviation industry. In this regard, the deformation of the tool is neglected. 

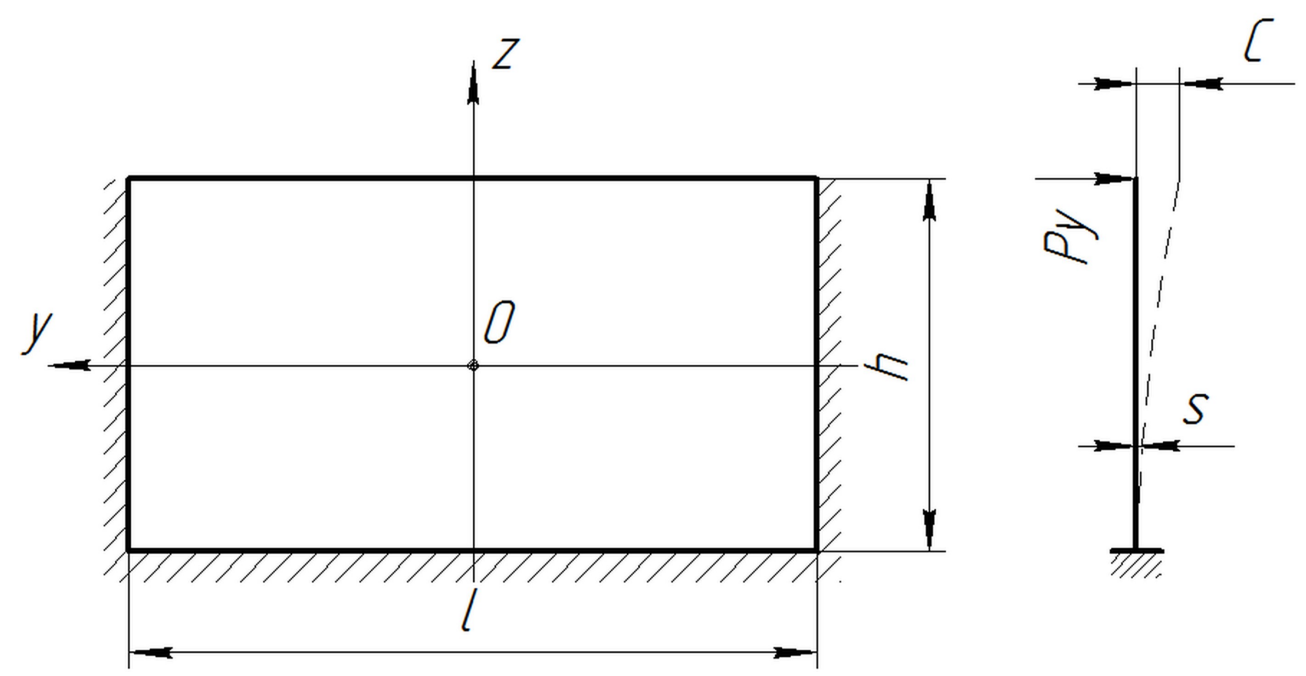

Fig. 2. Representation of the processed wall in the form of a plate.

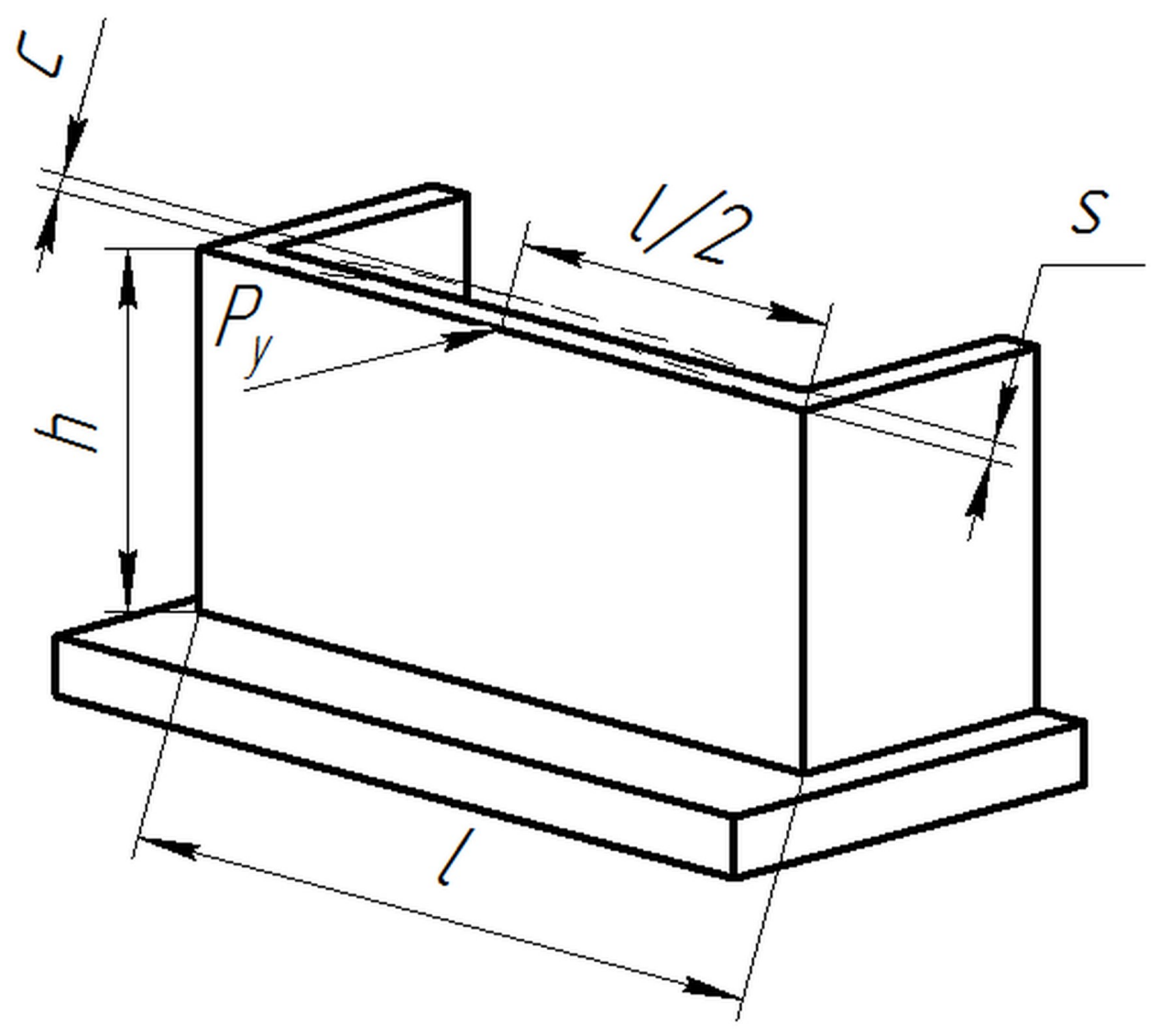

Fig. 3. Processed item. 
The maximum deviation of the wall $C$ from the specified position will be observed at the point as far as possible from all fixes, which is located in the middle of the processed element and at a distance $h$ from its base.

The calculation of the maximum elastic deformation of the wall is made according to the following formula:

$$
C=k_{W} \times \frac{P_{Y} \times h^{2}}{\pi D}
$$

$\mathbf{F}_{\mathrm{w}}$ - coefficient for the middle of the wall is equal to 0.527 ;

$\mathrm{P}_{\mathrm{y}}$ - radial component of cutting force, $\mathrm{N}$;

$\mathrm{h}$ - height of the wall, mm;

$\mathrm{D}$ - cylindrical wall stiffness, $\mathrm{kg} * \mathrm{~cm}$.

To check the efficiency of the given method, we will make a calculation. Consider the case: milled wall to which the sides adjacent to other structural elements. The billet of aluminum alloy D16T, the length of the wall $l=100 \mathrm{~mm}$, height $h=30 \mathrm{~mm}$, thickness $s=3$ $\mathrm{mm}$. Cutting tool - solid carbide mill of diameter $D=10 \mathrm{~mm}$, rotation speed $n=3000$ $\operatorname{Rev} / \mathrm{min}$, feed per tooth $S z=0.03 \mathrm{~mm} /$ tooth, cutting depth $T=5 \mathrm{~mm}$, width of cut $B=0.5$ $\mathrm{mm}$. Radial component of cutting force is equal to:

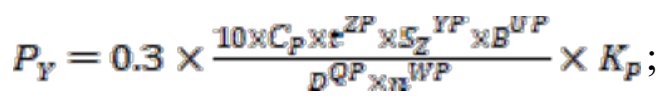

$$
\begin{aligned}
& P_{y}=0.3 \times \frac{10 \times 261 \times 5^{0.59} \times 0,03^{0.8} \times 0,5^{11.4}}{10^{1.41} \times 3000^{0.11}} \times 1.05=2.777 \mathrm{~N} \text {; }
\end{aligned}
$$

Using this value in the calculation of the Siemens NX CAE engineering analysis package, the following results were obtained: the maximum deviation $C$ of the wall was $0.0023 \mathrm{~mm}$.
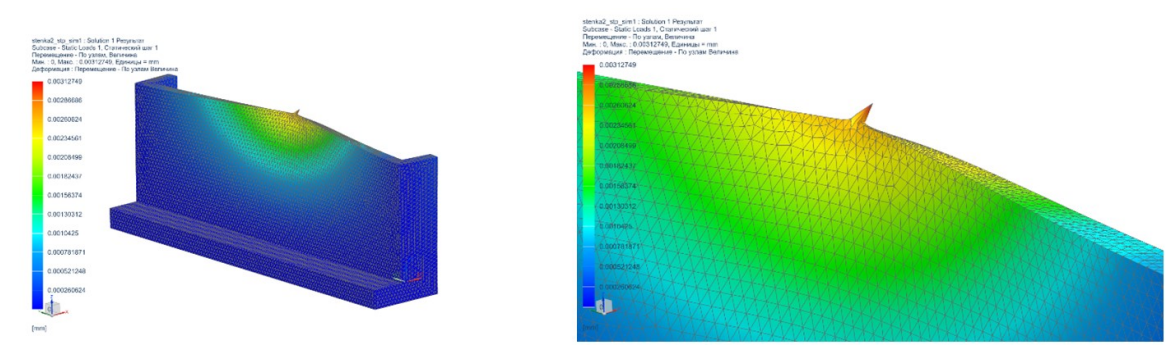

Fig. 4. Design model of the wall with adjacent structural elements.

Calculate the value of $C$ analytically:

$$
C=0.527 \frac{2.777 \times 30^{2}}{3.14 \times 174222.87}=0.0024 \mathrm{~mm}
$$

The difference between analytical and CAE calculations was $4 \%$. Thus, it can be concluded that the proposed mathematical model adequately reflects the research process.

\subsection{Calculation of cutting mode.}


To make a decision on the admissibility of the use of a milling mode, it is necessary to determine a number of restrictions: the maximum spindle speed, the maximum allowable elastic deformation of the workpiece, the highest performance.

The recommendations of the cutting tools manufacturers of a cutting speed are not always valid. Each machine has a value of the maximum spindle speed, which imposes restrictions on the maximum allowable cutting speed. In this regard, the speed values recommended by the cutting tools manufacturer are checked for applicability.

$$
n=\frac{1000 \times V}{\pi D} \leq n_{M A X} ;
$$

$\mathrm{n}$ - recommended frequency of rotation mill, rpm;

$\mathrm{n}_{\max }$ - maximum permissible speed, $\mathrm{rpm}$.

If the condition is not met, the maximum permissible value of the cutting speed $\mathrm{V}_{\max }$ is recalculated, which is used in further calculations.

$$
V_{\max }=\frac{\pi \times D \times n_{M A X}}{1000}, \mathrm{~m} / \mathrm{min}
$$

Consider the use of YG-1 Alu-Power cutter with a diameter of $10 \mathrm{~mm}$ when milling a workpiece is made of aluminum alloy D16T on the machining center DMG DMU 50 ecoline. Recommended cutting conditions for this mill: cutting speed $V=610 \mathrm{~m} / \mathrm{min}$, maximum feed per tooth $S z=0.13 \mathrm{~mm}$. the Maximum spindle speed of the selected machine $n_{\max }=12000 \mathrm{rpm}$.

$$
n=\frac{1000 \times 610}{3.14 \times 10}=19427 \mathrm{rpm} ;
$$

Recommended speed $19427 \mathrm{rpm}$ greater than the maximum permissible frequency of $12000 \mathrm{rpm}$. Determine the maximum cutting speed the permissible from machine characteristics.

$$
V_{\max }=\frac{3.14 \times 10 \times 12000}{1000}=376 \mathrm{~m} / \mathrm{min}
$$

For further calculations we accept $V_{\max } 376 \mathrm{~m} / \mathrm{min}$.

The following limiting condition is the maximum allowable elastic deformation of the wall $C_{\max }[6]$ :

$$
C_{\max }=\frac{d o p}{2} \times 0.8 \mathrm{~mm}
$$

dop - the maximum allowable deviation of the linear size of the processed element, $\mathrm{mm}$; 0.8 - factor, providing a margin of $20 \%$.

When processing the wall height $30 \mathrm{~h} 14 \mathrm{~mm}$, thickness $3 \mathrm{~h} 9\left(_{-0.025}\right) \mathrm{mm}$ determine the maximum permissible value of the wall elastic deformation:

$$
C_{\max }=\frac{0.025}{2} \times 0.8=0.01 \mathrm{~mm}
$$


The table of input data for finding a rational cutting mode is as follows:

Table 1. Input data for calculation

\begin{tabular}{|l|l|l|l|l|l|l|l|l|l|l|l|l|l|}
\hline $\mathbf{C p}$ & $\mathbf{D}$ & $\mathbf{n}$ & $\mathbf{K p}$ & $\mathbf{H B}$ & $\mathbf{z p}$ & $\mathbf{y p}$ & $\mathbf{u p}$ & $\mathbf{q p}$ & $\mathbf{w p}$ & $\mathbf{h}$ & $\mathbf{E}$ & $\mathbf{I}$ & $\mathbf{n p}$ \\
\hline 261 & 10 & 12000 & 1.053 & 200 & 0.9 & 0.8 & 1.1 & 1.1 & 0.13 & 30 & 69000 & 520.8 & 1 \\
\hline
\end{tabular}

$D$ - mill diameter, mm; $N$ - cutter speed, rpm; $C p$ - coefficient, reference data; zp, yp, up, qp, wp-exponents, reference data; $h$ - wall height, mm; $E$ - modulus of elasticity, MPa; $I-$ moment of inertia of the section, $\mathrm{mm}^{4}$.

The determination of the above conditions is followed by the calculation of all possible combinations of cutting parameters. For each set of parameters, the value of the radial component of the cutting force $P y$, the value of deformation $C$, as well as the amount of material removal per minute $Q$ is calculated.

$$
Q=\frac{T \times B \times S_{Z} \times n \times N_{Z}}{1000}, \mathrm{~cm}^{3} / \mathrm{min}
$$

$\mathbf{N z}$ - the number teeth of mill.

At this stage we vary the depth of milling $T$, the width of milling $B$, and the feed to the tooth $S z$. These parameters vary within the limits recommended by the mill manufacturer. Step variation for $S z-0.002 \mathrm{~mm} /$ tooth, for $T$ and $B-0.1 \mathrm{~mm}$.

For each step, the calculated wall deformation $C$ is compared with the allowable $C_{\max }$. If the condition $C \leq C_{\max }$ is not met, the data set is excluded from further calculation.

\section{Results and discussion}

The next step in determining the rational cutting mode is finding the most productive set of parameters. Table 2 shows an example of the calculated sets of elements of the cutting mode.

Table. 2. The result of the calculation (sampling)

\begin{tabular}{|c|c|c|c|c|c|}
\hline № & $\mathbf{B}, \mathrm{mm}$ & $\mathbf{T}, \mathrm{mm}$ & $\mathbf{S z}, \mathrm{mm} /$ tooth & $\mathbf{C}, \mathrm{mm}$ & $\mathbf{Q}, \mathrm{cm}^{3} / \mathrm{min}$ \\
\hline 1 & 1.0 & 10.0 & 0.015 & 0.0099 & 3.6 \\
\hline 2 & 1.0 & 7.8 & 0.027 & 0.0099 & 4.86 \\
\hline 3 & 1.0 & 5.4 & 0.054 & 0.0098 & 6.99 \\
\hline 4 & 1.0 & 3.8 & 0.120 & 0.0100 & 10.94 \\
\hline
\end{tabular}

Based on the calculation result, we obtain a set of elements of the most productive cutting mode: $V=376 \mathrm{~m} / \mathrm{min}, S z=0.12 \mathrm{~mm} /$ tooth, $T=3.8 \mathrm{~mm}, B=1 \mathrm{~mm}$, for which the value of the probable elastic wall deformation $C=0.01$ is known.

Thus, already at the stage of technological preparation of production, it is possible to adjust a tool path, which provides obtaining the dimensions of the processed element within the tolerance field specified by the drawing. In this example, it is recommended when using the found cutting mode to shift the trajectory by $0.01 \mathrm{~mm}$ to the treated surface (set a negative value of allowance), which will allow to obtain the thickness of the treated wall within $3_{-0.02} \mathrm{~mm}$, which eliminates the additional finishing pass required to remove the allowance remaining on the surface due to elastic deformations, the value of which exceeds the permissible values.

\section{Conclusion}


The method of determining the value of the machined element elastic deviation in the milling process is developed, as well as the method of using the calculation results in the appointment of a rational cutting mode.

\section{References}

1. Ezugwu, E \& Bonney, J \& Yamane, Yasuo, Journal of Materials Processing Technology, 134, 233 (2003)

2. M. Svinin, V. Savilov, IOP Conference Series: Materials Science and Engineering, 327, 314 (2018)

3. Campa Francisco, Lacalle Luis, Proceedings of the ASME International Manufacturing Science and Engineering Conference, 1011 (2008)

4. Qi Houjun, Tian Yanling, Zhang Dawei, The International Journal of Advanced Manufacturing Technology, 63 (2012)

5. Svetan Ratchev, Evan Govender, Journal of Materials Processing Technology, 143 (2003)

6. M.V. Nazarov, E.S. Kiselev, A.V. Popovich, MATEC Web of Conferences, 224, 1 (2018)

7. Antonialli Armando, Diniz Anselmo, Pederiva Robson. International Journal of Machine Tools and Manufacture, 50, 65 (2010)

8. B. Burdo. Russian Engineering Research, 37, 49 (2016)

9. V. Voronenko, Science intensive technologies in mechanical engineering, 45 (2018)

10. Ko Aung, Science intensive technologies in mechanical engineering, 21 (2019)

11. Xiao Gaobo, Ren Mingjun, To Suet. Micromachines, 9, 49 (2018)

12. Srichand Hinduja, Yongsheng Ma, Gibbou Barrow. International Journal of Machine Tools and Manufacture, 35, 689 (2015)

13. M Bogoljubova, A Afonasov, V Kozlov and O Sumtsova. IOP Conf. Series: Materials Science and Engineering, 124 (2016)

14. Alexey Popov, Sergei Babak. Manufacturing Technology, 18, 1011 (2018)

15. Yohanes Waloyo. AIP Conference Proceedings, 2097 (2019) 